\title{
Boron Nitride Nanotube as an Antimicrobial Peptide Carrier: A Theoretical Insight
}

\author{
Maryam Zarghami Dehaghani ${ }^{1}$ ** \\ Babak Bagheri ${ }^{2}$,* \\ Farrokh Yousefi $\mathbb{D D}^{3}$ \\ Abbasali Nasiriasayesh $^{4}$ \\ Amin Hamed Mashhadzadeh (iD) \\ Payam Zarrintaj ${ }^{6}$ \\ Navid Rabiee ${ }^{7}{ }^{7}$ \\ Mojtaba Bagherzadeh ${ }^{7}$ \\ Vanessa Fierro ${ }^{8}$ \\ Alain Celzard ${ }^{8}$ \\ Mohammad Reza Saeb ${ }^{5}$ \\ Ebrahim Mostafavi (iD) 9,10 \\ 'School of Chemical Engineering, College of \\ Engineering, University of Tehran, Tehran, \\ Iran; ${ }^{2}$ Department of Chemical and \\ Biomolecular Engineering, Korea Advanced \\ Institute of Science and Technology (KAIST), \\ Daejeon, Republic of Korea; ${ }^{3}$ Department of \\ Physics, University of Zanjan, Zanjan, 45I95- \\ 3/3, Iran; ${ }^{4}$ Industrial Management Institute, \\ Tehran, Iran; ${ }^{5}$ Center of Excellence in \\ Electrochemistry, School of Chemistry, \\ College of Science, University of Tehran, \\ Tehran, I4I55-6455, Iran; ${ }^{6}$ School of \\ Chemical Engineering, Oklahoma State \\ University, Stillwater, OK, 74078, USA; \\ ${ }^{7}$ Department of Chemistry, Sharif University \\ of Technology, Tehran, Iran; ${ }^{8}$ Université De \\ Lorraine, CNRS, IJL, Epinal, 88000, France; \\ 'Stanford Cardiovascular Institute, Stanford, \\ CA, USA; ${ }^{10}$ Department of Medicine, \\ Stanford University School of Medicine, \\ Stanford, CA, USA
}

*These authors contributed equally to this work

Correspondence: Ebrahim Mostafavi Stanford Cardiovascular Institute, Stanford University School of Medicine, Stanford, CA, USA

Email ebimsv@stanford.edu

Amin Hamed Mashhadzadeh

Center of Excellence in Electrochemistry,

School of Chemistry, College of Science,

University of Tehran, Tehran, 14I55-6455,

Iran

Email amin.hamed.m@gmail.com
This article was published in the following Dove Press journal:

International Journal of Nanomedicine
Introduction: Nanotube-based drug delivery systems have received considerable attention because of their large internal volume to encapsulate the drug and the ability to penetrate tissues, cells, and bacteria. In this regard, understanding the interaction between the drug and the nanotube to evaluate the encapsulation behavior of the drug in the nanotube is of crucial importance.

Methods: In this work, the encapsulation process of the cationic antimicrobial peptide named cRW3 in the biocompatible boron nitride nanotube (BNNT) was investigated under the Canonical ensemble (NVT) by molecular dynamics (MD) simulation.

Results: The peptide was absorbed into the BNNT by van der Waals (vdW) interaction between $\mathrm{cRW} 3$ and the BNNT, in which the $\mathrm{vdW}$ interaction decreased during the simulation process and reached the value of $-142.7 \mathrm{kcal} \cdot \mathrm{mol}^{-1}$ at $4 \mathrm{~ns}$.

Discussion: The increase in the potential mean force profile of the encapsulated peptide during the pulling process of cRW3 out of the nanotube showed that its insertion into the BNNT occurred spontaneously and that the inserted peptide had the desired stability. The energy barrier at the entrance of the BNNT caused a pause of $0.45 \mathrm{~ns}$ when half of the peptide was inside the BNNT during the encapsulation process. Therefore, during this period, the peptide experienced the weakest movement and the smallest conformational changes.

Keywords: boron nitride nanotube, drug delivery, antimicrobial peptide, molecular dynamic simulation, encapsulation

\section{Introduction}

Since the introduction of the first antimicrobial drug in the 1960s, penicillin, tremendous progress has been made in the treatment of infections. ${ }^{1}$ However, many obstacles have been encountered in the healing of microbial infections, even with the application of antimicrobial peptides. ${ }^{2}$ For example, pathogens are believed to be resistant to the specified antibiotic, making scientific research unprofitable. ${ }^{3}$ In this regard, the field of antimicrobial drug delivery has emerged to pave the way for the treatment of infections. ${ }^{4,5}$ Through drug delivery systems, the benefits of delivery are maintained and targeted to overcome side effects, ${ }^{6-9}$ delaying antimicrobial drug resistance, ${ }^{10,11}$ and protecting the drug against oxidation and catalysis by free radicals and enzymes, respectively. ${ }^{12,13}$

Nanoparticle-based antimicrobial drug delivery systems consist of biocompatible and non-toxic nanoparticles with a hydrodynamic diameter in the range of 10 to $100 \mathrm{~nm}$ that contain encapsulated or attached antimicrobial drugs. ${ }^{14-17}$ These drug 
delivery nano-systems are generally classified into six main groups, including solid nanoparticles, polymeric micelles, polymeric nanoparticles, liposomes, viral nanoparticles, and dendrimers. ${ }^{18-21}$ In addition to the mentioned advantages of drug delivery systems, nanocarriers would provide the benefits of a highly reactive area and the ability to penetrate cells, tissues, and bacteria. ${ }^{22-25}$

Nanotubes, as fascinating nanovectors, have attracted a lot of attention as drug carriers due to their distinct features such as having an inner volume large enough for drug encapsulation ${ }^{26}$ and surface functionalization. ${ }^{27-29}$ In recent decades, carbon nanotubes (CNTs) have been widely used as drug carriers. ${ }^{30}$ However, the possible cytotoxicity of CNTs has restricted their use in biomedical applications. ${ }^{30,31}$ Therefore, boron nitride nanotubes (BNNTs) have been introduced as alternatives because of their nontoxicity and biocompatibility due to their structural stability and chemical inertness. ${ }^{32-36}$ Moreover, because of their higher partial charges than CNTs, BNNTs demonstrate higher water permeation coefficients due to polarisation. ${ }^{37}$

In this respect, several types of research have been performed to investigate the performance of BNNTs as drug carriers. For example, Mortazavifar et $\mathrm{al}^{38}$ have verified the drug delivery performance of $\mathrm{OH}-$ functionalised BNNT using density functional theory (DFT) calculations and molecular dynamic (MD) simulations. According to the adsorption energy calculated by DFT, it was observed that the anticancer drug carmustine (CMT) was bound to the surface of the BNNT via hydrogen bonding with hydroxyl groups on the surface of the nanotube. In addition, they reported that increased CMT concentration and temperature resulted in a higher van der Waals force between $\mathrm{OH}-$ functionalised BNNT and the drug molecule. In another work by Roosta et al, ${ }^{39}$ the encapsulation of gemcitabine in $\operatorname{BNNT}(18,0)$ and the drug release performance using the heterofullerene $\left(\mathrm{C}_{48} \mathrm{~B}_{12}\right)$ releasing agent were studied using MD simulations. With regard to encapsulation, the drug was spontaneously located in the center of the BNNT over its entire length due to the negative interaction energy value of $-0.9 \mathrm{kcal} \mathrm{mol}^{-1}$. With regard to the release of the encapsulated drug, the $\mathrm{C}_{48} \mathrm{~B}_{12}$ releasing agent is added to the system and entered the BNNT, resulting in the release of the drug with a total potential energy difference of $-140.1 \mathrm{kcal}$ $\mathrm{mol}^{-1}$.
There are critical works in the literature regarding the use of different solvents for potential biomedical applications of BNNTs. Mirhaji et $\mathrm{al}^{40}$ verified the effect of the solvent containing water and ethanol on the encapsulation process of the anticancer drug docetaxel in a BNNT $(13,13)$ using MD simulations. According to the calculated van der Waals energies, solutions containing $60 \%$ and $75 \%$ ethanol had a negative effect on the interaction between docetaxel and BNNT. The penetration rate of the carboplatin drug in BNNT and BNNT having 18 hydroxyl groups on one edge was compared in the research of Khatti et al. ${ }^{41}$ It was observed that the hydroxyl groups facilitated the entry of the drug into the nanotube cavity. $\mathrm{Xu}$ et $\mathrm{al}^{42}$ compared the adsorption process of two anticancer drugs, temozolomide (TMZ) and CMT, in the presence of a BNNT $(6,6)$ as nanovector by DFT calculation. They reported that the energies of drug adsorption on the inner wall of the BNNT were remarkably higher than on the outer wall. Moreover, TMZ was absorbed on the surface of the nanotube with both $\pi-\pi$ and electrostatic interactions, which resulted in higher adsorption energy compared to the CMT/BNNT system with only electrostatic interactions.

MD simulation is considered a promising tool for predicting the performances of the systems containing nanostructures in various fields. ${ }^{43,44}$ In addition to evaluating the mechanical and thermal properties of nanomaterials, ${ }^{45-53}$ this computational approach provides the ability to evaluate the interatomic interactions in nanoparticle-based complex systems having biomolecules. $^{54}$ Therefore, in the current work, this method has been applied to study the atomic interactions and conformational changes of the peptide during the insertion process into the BNNT. According to previous research on the encapsulation of drugs in BNNT, the interaction between the nanotube and the drug is a key parameter in the encapsulation process. The drug should indeed pass the potential barrier at the nanotube entrance and, then stabilized via encapsulation process into the deep interior potential. Hence, to evaluate the performance of BNNT as a nanocarrier, it is very important to calculate the Van der Waals interaction between the drug and the nanotube during the encapsulation process, as well as the potential of mean force (PMF) of the encapsulated drug. In this manner, the selected drug, cRW3, is a small cationic antimicrobial peptide and has cyclic structure behavior in the 
BNNT, as nanocarrier, in an aqueous environment for drug delivery purposes. This effect has been verified by using MD simulations. Followed by that, the drug's encapsulation in the BNNT was evaluated by estimating the Van der Waals interaction between the drug and the BNNT. In addition, the stability of the drug in the BNNT was investigated by calculating the PMF of the encapsulated drug.

\section{Computational Details}

In the present work, the MD calculation was applied using the Large-Scale Atomic/Molecular Simulator (LAMMPS) software ${ }^{55}$ to verify the encapsulation process of the drug cRW3 in a boron nitride nanotube (BNNT) and, subsequently, the stability of the encapsulated drug in the nanotube. The visualization was obtained using VMD. ${ }^{56}$ In accordance with the size of cRW3, an armchair BNNT $(12,12)$ with a length of 30 $\dot{A}$ has been chosen as the nanocarrier for the drug. The axial direction of the nanotube was taken as the z-axis considered for the simulation box. The drug cRW3 was selected from the protein data bank (ID code 2OTQ). The chemical structure of cRW3 (cyclo-(Arg-Arg-TrpPhe-Trp-Arg)) is shown in Figure 1. The aromatic groups (tryptophane side chain) are responsible for the hydrophobic feature, while arginine groups are responsible for the hydrophilic part of cRW3 ${ }^{57,58}$

At the beginning of the simulation, cRW3 was placed at the initial distance of $2 \dot{\mathrm{A}}$ from the BNNT. The complex of BNNT and cRW3 was immersed in the simulation box consisting of TIP3P water molecules with the periodic boundary condition. Counter ions were then introduced into the simulation box to neutralize the simulated solution. Tersoff potential was applied to study the interaction between boron and nitrogen. ${ }^{59}$ All MD simulations were performed using the CHARMM27 force field. ${ }^{60}$ For the verification of the drug encapsulation process, first, the system was minimized in the NVT ensemble at 300 $\mathrm{K}$ while the BNNT was fixed. Second, the MD runs were performed in the NPT ensemble for 4 ns with a time step of $1 \mathrm{fs}$. For the vdW interaction, the inner and outer cut-off distances for the Lennard-Jones potential and Coulombic potential were 8 and $12 \dot{A}$, respectively. The LorentzBerthelot combination rule was applied to estimate the parameters of the Lennard-Jones potential for vdW interactions between non-bonded atoms. ${ }^{61}$ The vdW interaction between cRW3 and the BNNT can be obtained as: ${ }^{56}$

$$
E_{v d W-i n t}(t)=E_{c R W 3+B N N T}(t)-E_{c R W 3}(t)-E_{B N N T}(t)
$$

where $E_{v d W-i n t}$ is the vdW interaction between cRW3 and the BNNT, $E_{C R W 3+B N N T}$ corresponds to the vdW interaction of cRW3 combined with the BNNT, and $E_{c R W 3}$ and $E_{B N N T}$ refer to the vdW energies of cRW3 and the BNNT, respectively.

Regarding the stability of the drug encapsulated in the BNNT, an external force was applied to the encapsulated cRW3 along the z-axis of the nanotube to pull it out from the BNNT in the opposite direction to the penetrating process. The pulling velocity and spring constant $\mathrm{k}$ were $0.005 \dot{\mathrm{A} \mathrm{ps}}{ }^{-1}$ and $15 \mathrm{kcal} \mathrm{mol}^{-1} \dot{\mathrm{A}}^{-2}$, respectively. ${ }^{62}$ The pulling process was repeated ten times to obtain the potential of mean force (PMF) profile according to Jarzynski's equality: ${ }^{60}$

$$
e^{-\beta \Delta G}=e^{-\beta W}
$$

where $\Delta \mathrm{G}$ and $\mathrm{W}$ respectively represent the difference in free energy between two states and the work done on the system. $\beta$ is equal to $\left(K_{B} \mathrm{~T}\right)^{-1}$ where $K_{B}$ refers to the Boltzmann constant.

Langevin dynamics was considered to ensure $300 \mathrm{~K}$ as simulation temperature. The Langevin piston Nose'Hoover method was also used to fix the atmospheric pressure.

\section{Results and Discussion Localisation of cRW3 Within the Peptide-BNNT Complex}

The encapsulation of cRW3 into the BNNT $(12,12)$ was monitored through MD simulation, and snapshots taken by the VMD software at different times are shown in Figure 2. As can be seen, cRW3 was successfully inserted into the nanotube and remained stable up to 4 ns. Figure 3A shows the normalized center of mass (CoM) distance between the peptide and the BNNT, $\mathrm{d} / \mathrm{d}_{0}$ where $\mathrm{d}_{0}$ equals $23.43 \AA$, as a function of the simulation time. The fluctuation and the decrement of $\mathrm{d} / \mathrm{d}_{0}$ value at the beginning of the simulation confirmed that the peptide adjusted automatically during its entry into the BNNT. After the complete entry of the peptide into the nanotube cavity at $0.9 \mathrm{~ns}$, the CoM distance between the peptide and the BNNT remained approximately stable until the end of the simulation at 4 ns.

Due to the neutralized nature of the BNNT, the value of coulombic interactions (including hydrogen bonding, and dipole-dipole interactions) between the BNNT and the 


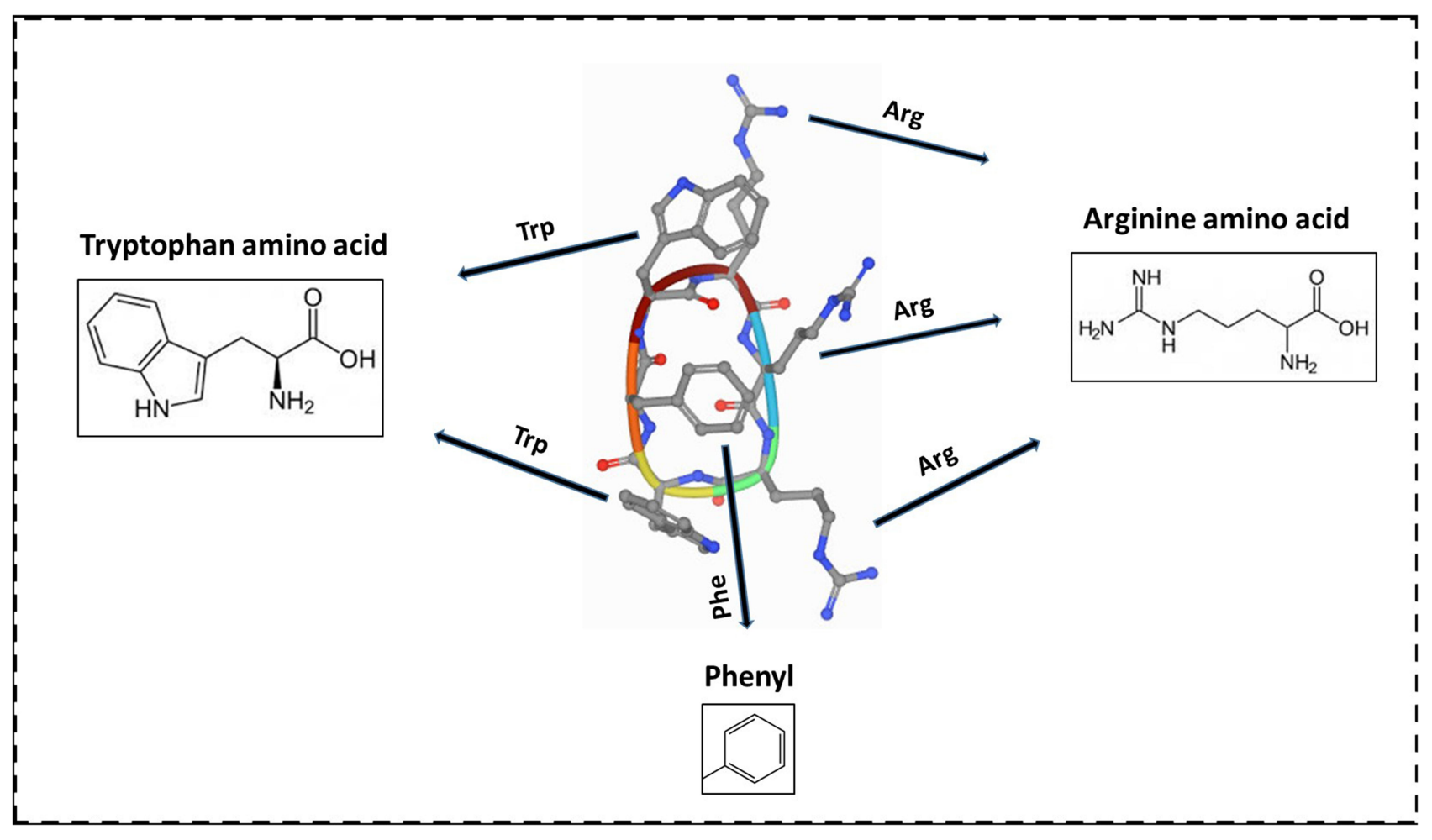

Figure I Amino acids at the basis of the chemical structure of the drug cRW3.

peptide was zero. Therefore, the vdW interaction energy (such as $\pi-\pi$ interactions between the aromatic rings of the peptide and the BNNT) is supposed as an absorbance energy of the peptide into the cavity of the BNNT.

For determining the role of the $\mathrm{vdW}$ interaction energy between the peptide and BNNT during the encapsulation process, the variation of the cRW3-BNNT vdW interaction energy with the simulation time was estimated, and the result was plotted as a curve shown in Figure 3B. As expected, the vdW interaction energy of the cRW3BNNT complex decreased with the decrement of $d / d_{0}$ value during the encapsulation process and reached the value of $-142.7 \mathrm{kcal} \cdot \mathrm{mol}^{-1}$ after the complete insertion of the peptide into the nanotube cavity at the time of $4 \mathrm{~ns}$. The same downward trend in the vdW energy of spontaneous insertion of DNA oligonucleotides and collagen-like peptides into CNTs has been reported by Huajian et $\mathrm{al}^{63}$ and Kang et al, ${ }^{64}$ respectively. In another research performed by Maleki et al, ${ }^{65}$ the vdW and electrostatic interactions between the Doxorubicin (DOX) and CNT were investigated. They demonstrated that due to the zero value of electro-static interactions, the absorbance of the DOX on the CNT was due to the vdW interactions with a favorable negative value.
The vdW interaction energy decreased rapidly until 0.34 ns when half of the peptide entered the BNNT. Then, for 0.45 ns, the peptide did not move in the nanotube and remained approximately static (with no decrease in CoM distance), and the peptide-BNNT vdW interaction energy did not change significantly. It can be explained that this phenomenon is related to the period when the peptide passed the maximum barrier energy at the entrance of the BNNT when half of the peptide was inserted inside the nanotube cavity. ${ }^{62}$ The yellow regions in Figure 3 illustrate this period of the encapsulation process. After crossing this energy barrier, the peptide was rapidly absorbed into the nanotube.

\section{Free Energy Calculation from MD Simulation}

After the complete insertion of cRW3 inside the BNNT at $4 \mathrm{~ns}$, the PMF profile of the encapsulated peptide was obtained by pulling it out through the MD simulation at the speed of $0.005 \dot{\mathrm{A}} \mathrm{ps}^{-1}$, which was chosen according to the speed of the encapsulation process. This simulation was repeated ten times to compute the average value of work (W) at each pulling distance, which is represented as a PMF profile in Figure 4. Moreover, the peptide positions along the $\mathrm{z}$-axis corresponding to the 


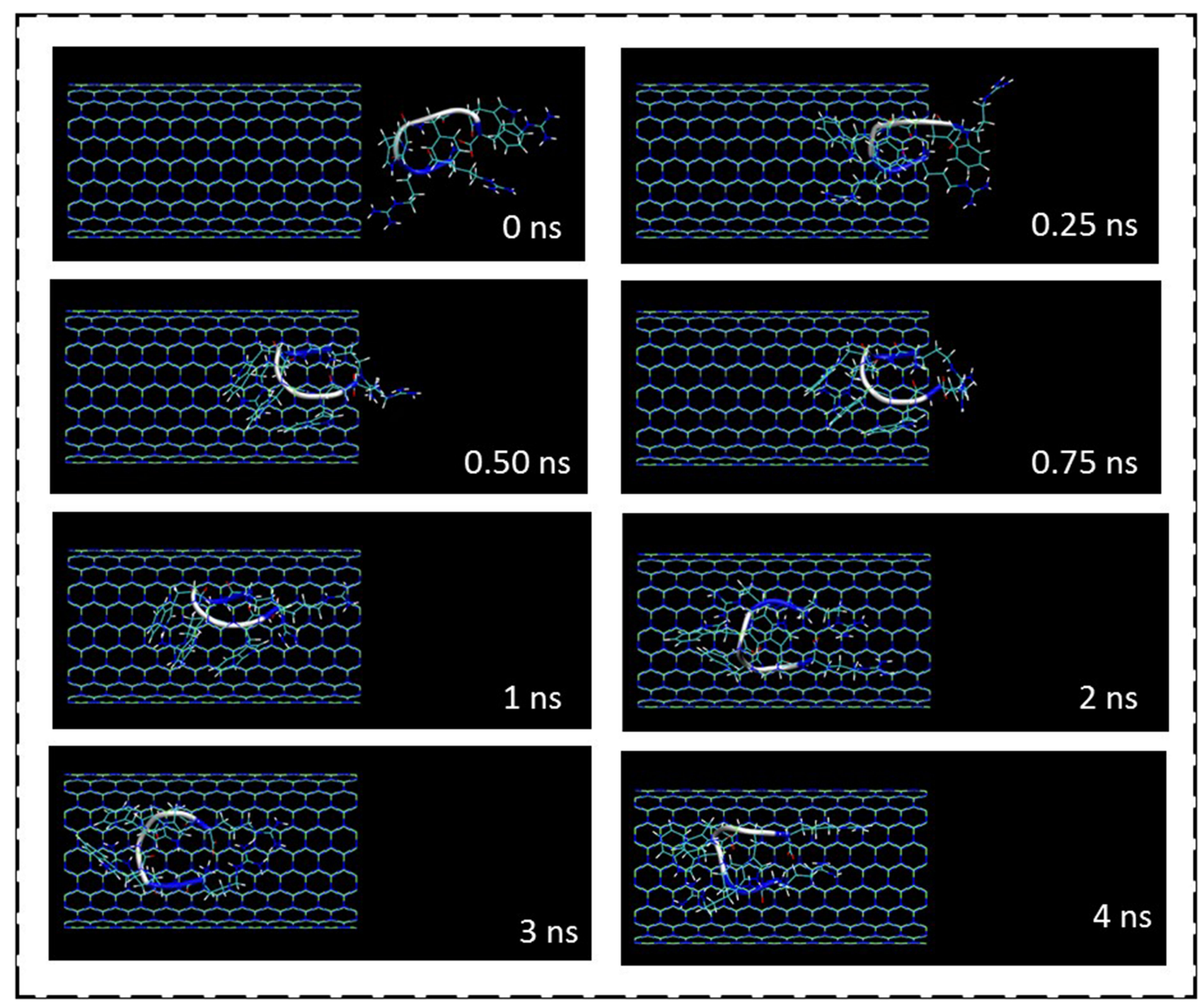

Figure 2 Representative snapshots of the insertion of the cationic antimicrobial peptide cRW3 into an armchair $($ I2, 12) BNNT at different times. For the sake of clarity, the water molecules are not shown.

BNNT are illustrated. It was observed that the free energy of this simulated system was increased during the pulling process and reached the value of 89.7 $\mathrm{kcal} \cdot \mathrm{mol}^{-1}$ at the pulling distance of $34 \AA$. This is in excellent agreement with the way the encapsulation process occurred spontaneously with the free energy of $-89.7 \mathrm{kcal} \cdot \mathrm{mol}^{-1}$. This observation is in good agreement with the results obtained in work done by Veclani et al ${ }^{66}$ so that the negative value of free energy $(\Delta G=-9.5$ $\mathrm{kcal} \cdot \mathrm{mol}^{-1}$ ) for the adsorption process of ciprofloxacin on the surface of CNT.

When half of the peptide entered the BNNT, the small barrier energy was detected in the PMF profile of the cRW3BNNT system, which is an enthalpic phenomenon.
A similar resistance corresponding to the potential energy barrier at the entrance of the carbon nanotube during the insertion of water molecules was reported. ${ }^{67}$ It was explained that the peptide was absorbed toward the BNNT cavity due to the vdW interaction, which overcame the hydrogen bonding between the water molecules. By decreasing the distance between the BNNT and cRW3, the higher accumulation of hydrogen bonding networks near and within the BNNT resulted in a $0.45 \mathrm{~ns}$ pause during the encapsulation process. However, the cRTW3-BNNT vdW interaction destroyed these hydrogen-bonding networks and overcame the energy barrier, which then allowed the favorable encapsulation process of the peptide inside the nanotube. ${ }^{62}$ 

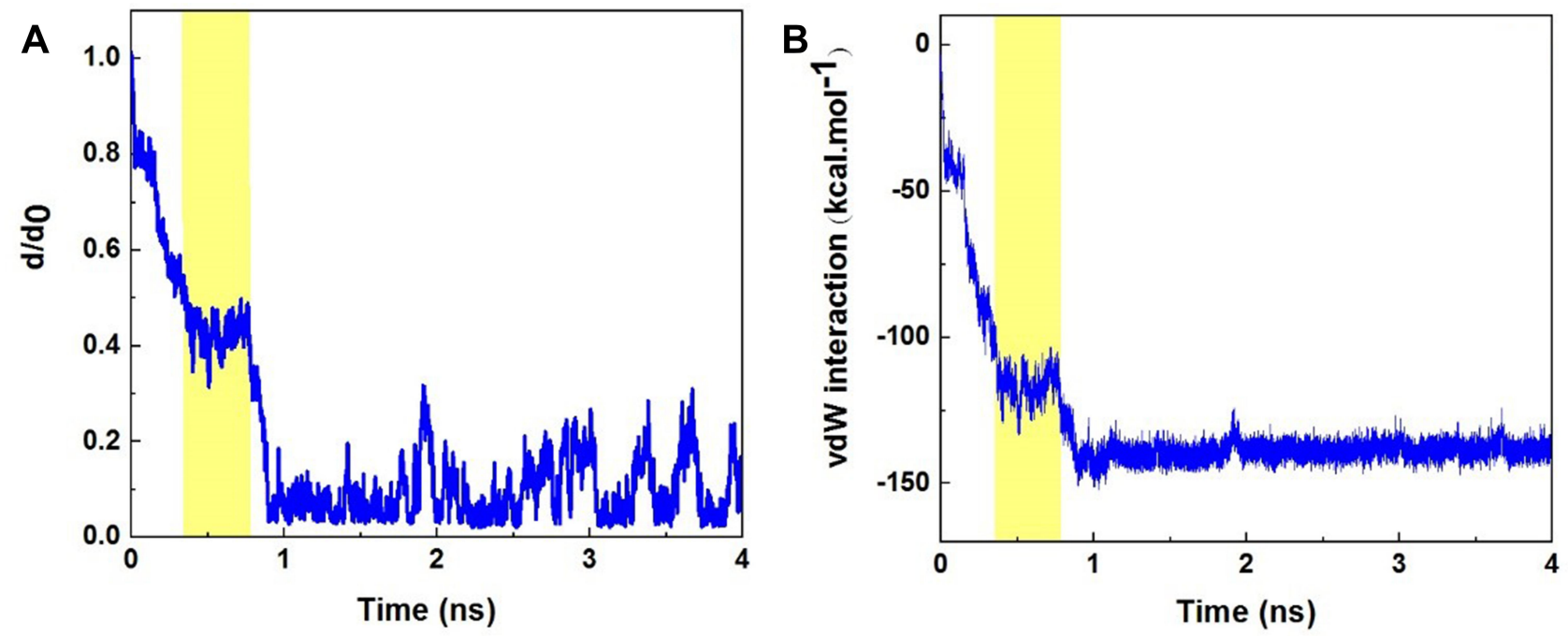

Figure 3 (A) $d / d_{0}$ (normalized CoM distance where $d_{0}$ is the initial CoM distance) between the cationic antimicrobial peptide cRW3 and the boron nitride nanotube as a function of simulation time; $(B) v d W$ interaction between cRW3 and the BNNT $(12,12)$ as a function of simulation time. The yellow regions illustrate the encapsulation period.

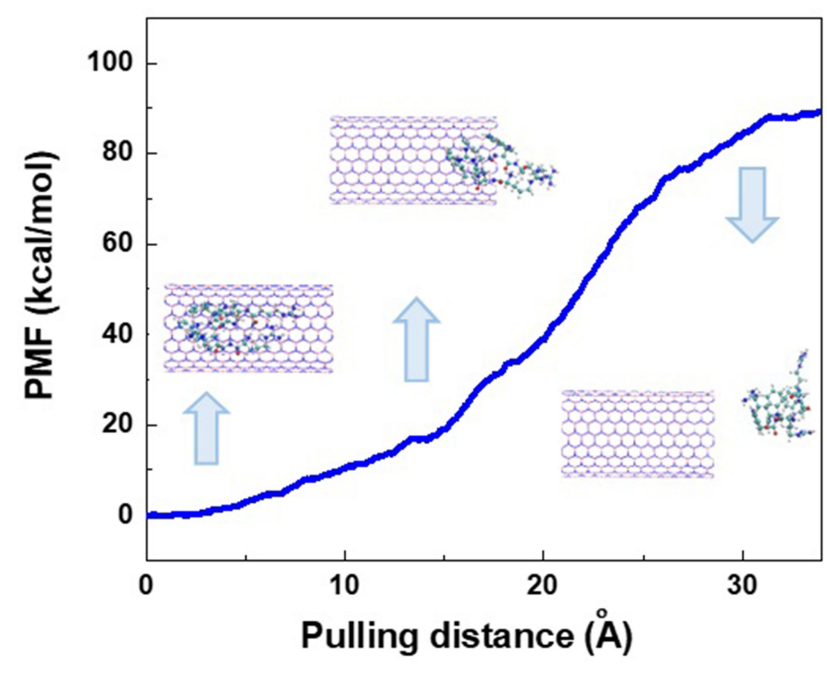

Figure 4 Potential of mean force (PMF) computed from ten pullings by MD simulation. The images represent the positions of the cationic antimicrobial peptide cRW3 corresponding to the z-coordinate along the BNNT at some key position.

\section{Variations in the Conformation of the Peptide}

Figure 5A depicts the changes in the peptide structure at 0 and $4 \mathrm{~ns}$ of MD simulation. The left image of Figure 5A is attributed to the peptide immersed in water molecules in its natural form at $0 \mathrm{~ns}$. The image on the right represents the conformation of the peptide adjusted to the BNNT structure at $4 \mathrm{~ns}$, demonstrating that the conformation of the peptide has adapted to the geometry of the BNNT after its complete insertion in the cavity of the nanotube. Similar changes in the configuration and arrangement of
DOX encapsulated within the single-walled carbon nanotube in accordance with the diameter and chirality of the nanotube have been reported by Zhang et al. ${ }^{68}$

Figure 5B shows the root mean square deviation (RMSD), which reveals the variations in the peptide conformation as a function of simulation time. As seen in this figure, the RMSD varied significantly by $0.34 \mathrm{~ns}$, showing that changes in the peptide conformation are affected by the high cRW3-BNNT vdW interaction. The yellow region corresponds to the time during which half of the peptide was inserted into the BNNT, and the encapsulation process was interrupted for 0.45 ns. It was observed that, during this period, not only did the peptide not move toward the BNNT, but also that the changes in the configuration of the peptide were not significant, which is due to the energy barrier in the vicinity of the BNNT. However, after that, the RMSD fluctuated within the specified range, which shows the self-adjustment of the peptide to the inner geometry of the BNNT. Moreover, due to the small size and cyclic structure of the peptide, all hydrophobic and hydrophilic parts were equally influenced by the hydrophilic solvent and the hydrophobic nanotube, so that the distinct phase separation between hydrophobic and hydrophilic groups was not observed. Nevertheless, the different affinity of the hydrophobic and the hydrophilic groups for the solvent and the nanotube would be observed in the system containing larger biomolecules such as proteins. ${ }^{69}$

Considering the changes in the gyration radius of the peptide during the encapsulation process (Figure 5C), it was 

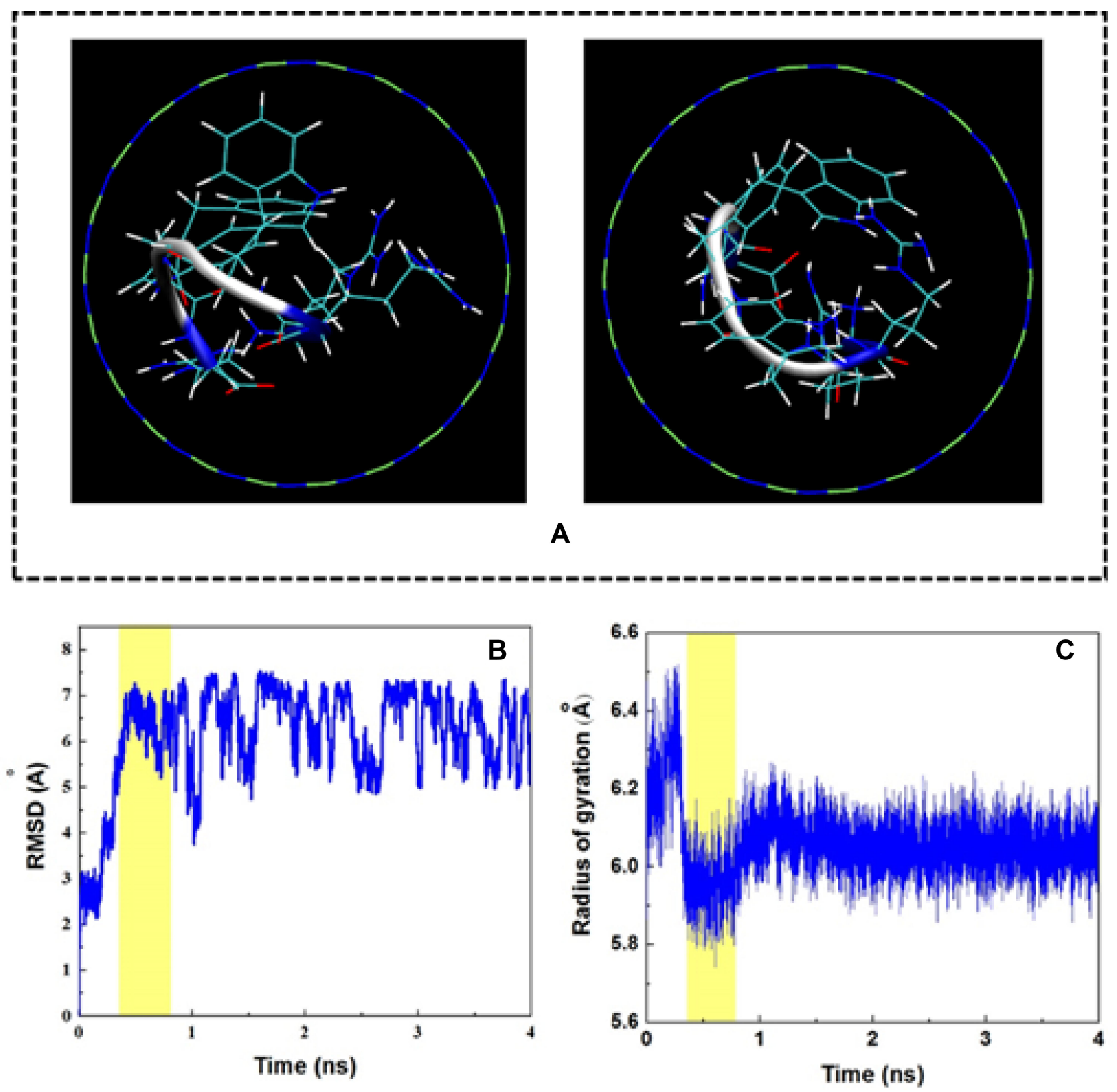

Figure 5 (A) Axial views of the cationic antimicrobial peptide cRW3 at 0 ns and 4 ns in the MD simulation. For clarity, water molecules were not shown. (B) Root mean square deviation (RMSD) of cRW3 as a function of simulation time. (C) Gyration radius of cRW3 as a function of simulation time. The yellow regions illustrate the encapsulation period.

observed that the peptide was stretched by the cRTW3BNNT vdW interaction until 0.34 ns of simulation time, which resulted in an increase in the gyration radius of the peptide. Then, for a period of $0.45 \mathrm{~s}$ (the yellow region in which half of the peptide was in the BNNT), the gyration radius reached its minimum value, showing the adjustment process during which the size of the peptide changed to match the movement towards the entrance hole of the BNNT. Subsequently, the configuration of the peptide changed to a more stable state with a higher gyration radius of about $6.1 \AA$. The variation in the distance between the central axis of the BNNT and the CoM of the peptide cRW3 during the simulation process is shown in Figure $6 \mathrm{~A}$. At $0 \mathrm{~ns}$, the peptide was located at the specified location in the simulation box so that the CoM of the peptide was adjusted on the central axis of the BNNT. The peptide was then transferred to one side of the BNNT wall in relation to the initial reorientation of the peptide by the $\mathrm{vdW}$ interaction so that the cRW3-axis distance increased to $2.6 \AA$ at $0.11 \mathrm{~ns}$. However, this conformation of the peptide was also altered 

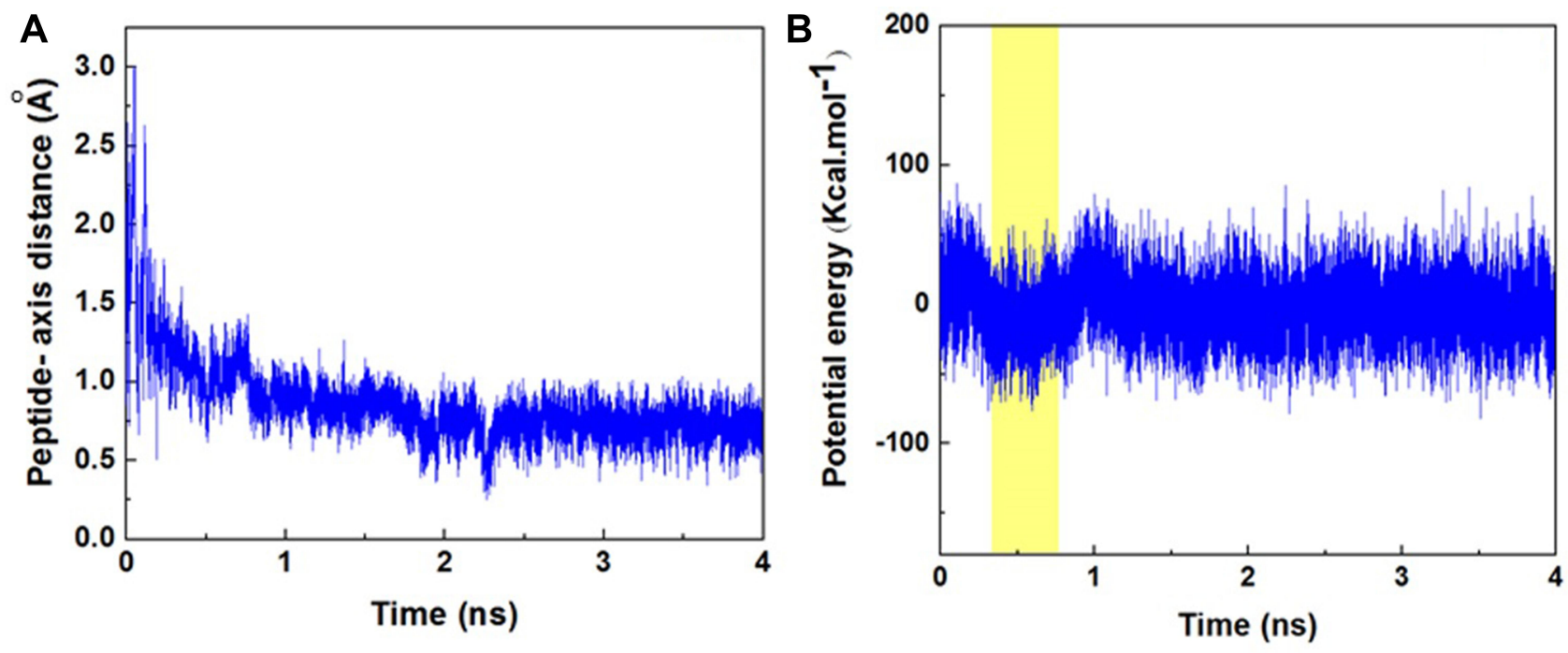

Figure 6 (A) Distance between the CoM of cRW3 and the central axis of the BNNT; and (B) potential energy of cRW3 as a function of simulation time, both as a function of simulation time. The yellow region illustrates the encapsulation period.

by adsorption to other sidewalls of the BNNT, which then probably caused the cRW3-axis distance to decrease. In other words, the hydrophobic groups in the structure of the peptide are strongly affected by the vdW interaction between the BNNT and the peptide, so that the CoM location of the peptide alternately changed towards the sidewall of the nanotube, appearing as the fluctuation of the cRW3axis distance curve during the encapsulation procedure.

Figure $6 \mathrm{~A}$ and $\mathrm{B}$ illustrates the potential energy changes of cRW3 during the simulation process. As can be seen, the potential energy of cRW3 was increased undesirably at the beginning of the encapsulation process, corresponding to a low-stability conformation. During the period of time when half of the peptide was placed in the BBNT cavity, and the pause in the encapsulation process occurred (the yellow region in Figure $6 \mathrm{~B}$ ), the potential energy of the peptide reached its minimum value, which is favorable for the continuation of the insertion process after this pause in the encapsulation process. The decrease in the potential energy of the peptide was due to the adjustment of the conformation of the peptide and was compensated by the energy of the system. After this time, the cRTW3-BNNT vdW interaction overcame the energy barrier, and the potential energy of the peptide increased again. The potential energy fluctuation of the peptide completely inserted into the BNNT was attributed to the continuous changes in conformation, as discussed above.

For a deeper understanding of the conformational changes (self-adjustment) of the peptide during the encapsulation process, especially during the time period in which the peptide was entering the cavity of the BNNT, the distance of each residue in the structure of the peptide cRTW3 from the central axis of the BNNT was calculated. As can be seen in Figure 7, at the beginning of the simulation, the variations in the distances of residues from the central axis of the BNNT were intensive, corresponding to the movement freedom of the peptide structure in its natural state. However, by approaching the peptide to the cavity of the BNNT, these changes became more smooth in a way that at the end of yellow region, where half of the peptide was inserted inside the BNNT, up to the end of the simulation all distances fluctuated in the narrow regions. These observations confirmed the stability of the encapsulated peptide under the effect of vdW interaction energy between the peptide residues and the interior wall of the BNNT.

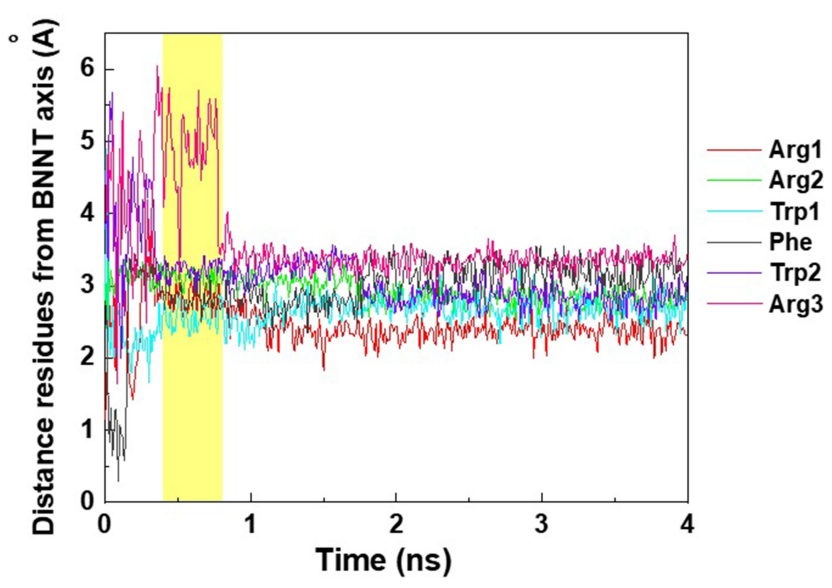

Figure 7 The distance of residues in the structure of the drug CRW3 from the central axis of the BNNT $(20,20)$. The yellow regions illustrate the encapsulation period. 
In other words, the conformational changes of the peptide residues within the yellow region made a great contribution to the encapsulation process so that the peptide could pass the energy barrier in the vicinity of the BNNT. During this period: 1) the peptide did not move in the direction toward the cavity of the BNNT, 2) the vdW energy between the BNNT and the peptide did not experience the reduction, 3) the potential energy of the peptide decreased in favour of the insertion process, 4) the gyration radius of the peptide reached the minimum, 5) the changes of the RMSD were not significant. At the end of this period, the peptide was appropriately adjusted to the geometry of the interior wall of the BNNT associated with the minimum value of the potential and vdW energies which confirmed the stability of the encapsulated peptide.

\section{Conclusion}

In the present work, the encapsulation process of the antimicrobial peptide cRW3 in a boron nitride nanotube (BNNT) in an aqueous medium was explored by MD simulation. It was observed that the peptide-BNNT vdW interaction energy and the normalized center of mass (CoM) distance between the peptide and the BNNT, $\mathrm{d} / \mathrm{d}_{0}$, decreased during the encapsulation process and reached values of $-142.7 \mathrm{kcal} \cdot \mathrm{mol}^{-1}$ and 0 , respectively. The calculation of the potential mean force, which was performed through a pulling process, revealed that the encapsulation process occurred spontaneously with the free energy of $-89.7 \mathrm{kcal} \cdot \mathrm{mol}^{-1}$. For a period of $0.45 \mathrm{~ns}$, when half of the peptide was inserted into the BNNT cavity, the encapsulation process was interrupted due to the energy barrier caused by the hydrogen bonding networks accumulated in the vicinity of the nanotube. During this period, it was revealed that:

1. $d / d_{0}$ and the vdW interaction energy between the peptide and the BNNT did not change significantly.

2. Based on the changes in root mean square deviation and gyration radius of the peptide, the latter did not undergo significant conformational changes and had the lowest gyration radius.

3. The decrease in the potential energy of the peptide occurred due to the conformational adjustment of the peptide.

BNNT possesses promising features such as large internal volume, ability to penetration into tissues, biocompatibility, chemical inertness, non-toxicity, and acceptable water solubility, which makes it newly emerged nanocarriers in drug-based therapies. In addition, in accordance with obtained results, it was demonstrated that the cationic antimicrobial peptide cRW3 was successfully encapsulated spontaneously into BNNT through the desired vdW interactions between the peptide and the BNNT. Owing to the aforementioned factors, the BNNT-based drug delivery systems can be considered as an effective approach to delaying antimicrobial drug resistance. As a result, there is a need for further investigation on the performance of BNNT both theoretically and experimentally to achieve significant progress in the field of nanomedicine.

\section{Data Sharing Statement}

All data reported here can be made available on request by the corresponding authors.

\section{Disclosure}

The authors report no conflicts of interest in this work.

\section{References}

1. Coates A, Hu Y, Bax R, Page C. The future challenges facing the development of new antimicrobial drugs. Nat Rev Drug Discov. 2002;1(11):895-910. doi:10.1038/nrd940

2. Seo M-D, Won H-S, Kim J-H, Mishig-Ochir T, Lee B-J. Antimicrobial peptides for therapeutic applications: a review. Molecules. 2012;17(10):12276-12286. doi:10.3390/molecules17 1012276

3. Pham TN, Loupias P, Dassonville-Klimpt A, Sonnet P. Drug delivery systems designed to overcome antimicrobial resistance. Med Res Rev. 2019;39(6):2343-2396. doi:10.1002/med.21588

4. Nandi SK, Mukherjee P, Roy S, Kundu B, De DK, Basu D. Local antibiotic delivery systems for the treatment of osteomyelitis a review. Mater Sci Eng C. 2009;29(8):2478-2485. doi:10.1016/j. msec.2009.07.014

5. Yazdi MK, Taghizadeh A, Taghizadeh M, et al. Agarose-based biomaterials for advanced drug delivery. J Control Release. 2020.

6. Zhang Y, Zhang J, Chen M, et al. A bioadhesive nanoparticlehydrogel hybrid system for localized antimicrobial drug delivery. ACS Appl Mater Interfaces. 2016;8(28):18367-18374. doi:10.1021/ acsami.6b04858

7. Moseke C, Hage F, Vorndran E, Gbureck U. TiO2 nanotube arrays deposited on Ti substrate by anodic oxidation and their potential as a long-term drug delivery system for antimicrobial agents. APPL SURF SCI. 2012;258(14):5399-5404. doi:10.1016/j.apsusc.2012.02.022

8. Pishnamazi M, Hafizi H, Pishnamazi M, Marjani A, Shirazian S, Walker GM. Controlled release evaluation of paracetamol loaded amine functionalized mesoporous silica $\mathrm{KCC} 1$ compared to microcrystalline cellulose based tablets. Sci Rep. 2021;11(1):535. doi:10.1038/s41598-020-79983-8

9. Li R, Wang Y, Du J, et al. Graphene oxide loaded with tumor-targeted peptide and anti-cancer drugs for cancer target therapy. Sci Rep. 2021;11(1):1725. doi:10.1038/s41598-021-81218-3

10. Smith AW. Biofilms and antibiotic therapy: is there a role for combating bacterial resistance by the use of novel drug delivery systems? Adv Drug Deliv Rev. 2005;57(10):1539-1550. doi:10.1016/j. addr.2005.04.007 
11. Zarrintaj P, Ramsey JD, Samadi A, et al. Poloxamer: a versatile tri-block copolymer for biomedical applications. Acta Biomaterialia. 2020.

12. Bernkop-Schnürch A, Bratengeyer I, Valenta C. Development and in vitro evaluation of a drug delivery system protecting from trypsinic degradation. Int J Pharm. 1997;157(1):17-25. doi:10.1016/S03785173(97)00198-1

13. Poon W, Kingston BR, Ouyang B, Ngo W, Chan WCW. A framework for designing delivery systems. Nat Nanotechnol. 2020;15(10):819-829. doi:10.1038/s41565-020-0759-5

14. Wilczewska AZ, Niemirowicz K, Markiewicz KH, Car $\mathrm{H}$. Nanoparticles as drug delivery systems. Pharmacol Rep. 2012;64 (5):1020-1037. doi:10.1016/S1734-1140(12)70901-5

15. Gao W, Chen Y, Zhang Y, Zhang Q, Zhang L. Nanoparticle-based local antimicrobial drug delivery. Adv Drug Deliv Rev. 2018;127:46-57. doi:10.1016/j.addr.2017.09.015

16. Babanejad N, Nabid MR, Farhadian A, et al. Sustained delivery of olanzapine from sunflower oil-based polyol-urethane nanoparticles synthesised through a cyclic carbonate ring-opening reaction. IET Nanobiotechnol. 2019;13(7):703-711. doi:10.1049/iet-nbt.2018.5440

17. Mitchell MJ, Billingsley MM, Haley RM, Wechsler ME, Peppas NA, Langer R. Engineering precision nanoparticles for drug delivery. Nat Rev Drug Discov. 2020;20:101-124. doi:10.1038/s41573-020-0090-8

18. Cho K, Wang X, Nie S, Shin DM, Shin DM. Therapeutic nanoparticles for drug delivery in cancer. Clin Cancer Res. 2008;14 (5):1310-1316. doi:10.1158/1078-0432.CCR-07-1441

19. Zarrintaj P, Yazdi MK, Bagheri B, Kim YC, Ramsey JD, Saeb MR. Block copolymers for nanoscale drug and gene delivery. Nanoeng Biomater Adv Drug Deliv. 2020;181-200.

20. Yazdi MK, Zarrintaj P, Bagheri B, Kim YC, Ganjali MR, Saeb MR. Nanotechnology-based biosensors in drug delivery. Nanoeng Biomater Adv Drug Deliv. 2020;767-779.

21. Yazdia MK, Zarrintajb P, Ghavamic M, Alizadehd R, Saebe MR. Protein and peptide-based delivery systems. Nanoeng Biomater Adv Drug Deliv. 2020;145.

22. Ramos MADS, Da Silva PB, Spósito L, et al. Nanotechnology-based drug delivery systems for control of microbial biofilms: a review. Int J Nanomedicine. 2018;13:1179. doi:10.2147/IJN.S146195

23. Servatan M, Zarrintaj P, Mahmodi G, et al. Zeolites in drug delivery: progress, challenges and opportunities. Drug Discov Today. 2020;25 (4):642-656. doi:10.1016/j.drudis.2020.02.005

24. Zarrintaj P, Mahmodi G, Manouchehri S, et al. Zeolite in tissue engineering: opportunities and challenges. MedComm. 2020;1 (1):5-34. doi:10.1002/mco2.5

25. Singh AP, Biswas A, Shukla A, Maiti P. Targeted therapy in chronic diseases using nanomaterial-based drug delivery vehicles. Signal Transduct Target Ther. 2019;4(1):33.

26. Arsawang U, Saengsawang O, Rungrotmongkol T, et al. How do carbon nanotubes serve as carriers for gemcitabine transport in a drug delivery system? J Mol Graph Model. 2011;29(5):591-596. doi:10.1016/j.jmgm.2010.11.002

27. Yoosefian M, Etminan N. Density functional theory (DFT) study of a new novel bionanosensor hybrid; tryptophan/Pd doped single walled carbon nanotube. Physica E Low Dimens Syst Nanostruct. 2016;81:116-121. doi:10.1016/j.physe.2016.03.009

28. Yazdi MK, Saeidi H, Zarrintaj P, Saeb MR, Mozafari M. PANI-CNT nanocomposites. Fundament Emerg Appl Polyaniline. 2019;143-163.

29. Jouyandeh M, Tikhani F, Hampp N, et al. Highly curable self-healing vitrimer-like cellulose-modified halloysite nanotube/epoxy nanocomposite coatings. Chem Eng J. 2020;396:125196. doi:10.1016/j. cej.2020.125196

30. Bottini M, Bruckner S, Nika K, et al. Multi-walled carbon nanotubes induce T lymphocyte apoptosis. Toxicol Lett. 2006;160(2):121-126. doi:10.1016/j.toxlet.2005.06.020

31. Lewinski N, Colvin V, Drezek R. Cytotoxicity of nanoparticles. small. 2008;4(1):26-49. doi:10.1002/smll.200700595
32. Chen X, Wu P, Rousseas $\mathrm{M}$, et al. Boron nitride nanotubes are noncytotoxic and can be functionalized for interaction with proteins and cells. J Am Chem Soc. 2009;131(3):890-891. doi:10.1021/ ja807334b

33. Cohen ML, Zettl A. The physics of boron nitride nanotubes. Phys Today. 2010;63(11):34-38. doi:10.1063/1.3518210

34. Zarghami Dehaghani M, Bagheri B, Nasiriasayesh A, et al. Insight into the self-insertion of a protein inside the boron nitride nanotube. ACS Omega. 2020;5:32051-32058. doi:10.1021/acsomega.0c05080

35. Allard C, Schué L, Fossard F, et al. Confinement of dyes inside boron nitride nanotubes: photostable and shifted fluorescence down to the near infrared. Adv Mater. 2020;32:2001429. doi:10.1002/ adma.202001429

36. Niskanen J, Zhang I, Xue Y, Golberg D, Maysinger D, Winnik FM. Boron nitride nanotubes as vehicles for intracellular delivery of fluorescent drugs and probes. Nanomedicine. 2016;11(5):447-463. doi: $10.2217 / \mathrm{nnm} .15 .214$

37. Won CY, Aluru NR. Structure and dynamics of water confined in a boron nitride nanotube. J Phys Chem C. 2008;112(6):1812-1818. doi:10.1021/jp076747u

38. Mortazavifar A, Raissi H, Akbari A. DFT and MD investigations on the functionalized boron nitride nanotube as an effective drug delivery carrier for Carmustine anticancer drug. $J$ Mol Liq. 2019;276:577-587. doi:10.1016/j.molliq.2018.12.028

39. Roosta S, Nikkhah SJ, Sabzali M, Hashemianzadeh SM. Molecular dynamics simulation study of boron-nitride nanotubes as a drug carrier: from encapsulation to releasing. RSC Adv. 2016;6 (11):9344-9351. doi:10.1039/C5RA22945F

40. Mirhaji E, Afshar M, Rezvani S, Yoosefian M. Boron nitride nanotubes as a nanotransporter for anti-cancer docetaxel drug in water/ ethanol solution. J Mol Liq. 2018;271:151-156. doi:10.1016/j. molliq.2018.08.142

41. Khatti Z, Hashemianzadeh SM. Boron nitride nanotube as a delivery system for platinum drugs: drug encapsulation and diffusion coefficient prediction. Eur J Pharm Sci. 2016;88:291-297. doi:10.1016/j. ejps.2016.04.011

42. Xu H, Wang Q, Fan G, Chu X. Theoretical study of boron nitride nanotubes as drug delivery vehicles of some anticancer drugs. Theor Chem Acc. 2018;137(7):104. doi:10.1007/s00214-018-2284-2

43. Katiyar RS, Jha PK. Molecular simulations in drug delivery: opportunities and challenges. Wiley Interdiscip Rev Comput Mol Sci. 2018;8(4):e1358.

44. Singh A, Vanga SK, Orsat V, Raghavan V. Application of molecular dynamic simulation to study food proteins: a review. Crit Rev Food Sci Nutr. 2018;58(16):2779-2789. doi:10.1080/10408398.201 7.1341864

45. Bagheri B, Dehaghani MZ, Karami Z, et al. Correlation between surface topological defects and fracture mechanism of $\gamma$-graphynelike boron nitride nanosheets. Comput Mater Sci. 2020;110152.

46. Salmankhani A, Karami Z, Mashhadzadeh AH, et al. A theoretical scenario for the mechanical failure of boron carbide nanotubes. Comput Mater Sci. 1100;186:22.

47. Mashhadzadeh AH, Taghizadeh A, Taghizadeh M, et al. MetalOrganic Framework (MOF) through the lens of molecular dynamics simulation: current status and future perspective. J Compos Sci. 2020;4(2):75. doi: $10.3390 /$ jcs4020075

48. Dehaghani MZ, Mashhadzadeh AH, Salmankhani A, et al. Fracture toughness and crack propagation behavior of nanoscale beryllium oxide graphene-like structures: a molecular dynamics simulation analysis. Eng Fract Mech. 2020;235:107194. doi:10.1016/j. engfracmech.2020.107194

49. Ahangari MG, Mashhadzadeh AH. Density functional theory based molecular dynamics study on hydrogen storage capacity of $\mathrm{C} 24$, $\mathrm{B} 12 \mathrm{~N} 12$, Al12 N12, Be12O12, Mg12O12, and $\mathrm{Zn} 12 \mathrm{O} 12$ nanocages. Int $J$ Hydrogen Energy. 2020;45(11):6745-6756. doi:10.1016/j.ijhydene.2019.12.106 
50. Salmankhani A, Karami Z, Mashhadzadeh AH, Saeb MR, Fierro V, Celzard A. Mechanical properties of C3N nanotubes from molecular dynamics simulation studies. Nanomaterials. 2020;10(5):894. doi:10.3390/nano10050894

51. Albooyeh A, Dadrasi A, Mashhadzadeh AH. Effect of point defects and low-density carbon-doped on mechanical properties of BNNTs: a molecular dynamics study. Mater Chem Phys. 2020;239:122107. doi:10.1016/j.matchemphys.2019.122107

52. Dadrasi A, Albooyeh A, Mashhadzadeh AH. Mechanical properties of silicon-germanium nanotubes: a molecular dynamics study. Appl Surf Sci. 2019;498:143867. doi:10.1016/j.apsusc.2019.143867

53. Zarghami Dehaghani M, Salmankhani A, Hamed Mashhadzadeh A, Habibzadeh S, Abida O, Reza Saeb M. Fracture mechanics of polycrystalline beryllium oxide nanosheets: a theoretical basis. Eng Fract Mech. 2021;244:107552. doi:10.1016/j.engfracmech.2021.107552

54. Fatemi SM, Foroutan M. Review of recent studies on interactions between polymers and nanotubes using molecular dynamic simulation. J Iran Chem Soc. 2017;14(2):269-283. doi:10.1007/ s13738-016-0976-x

55. Fast Parallel PS. Algorithms for short-range molecular dynamics. J Comput Phys. 1995;117(1):1-19. doi:10.1006/jcph.1995.1039

56. Humphrey W, Dalke A, Schulten K. VMD: visual molecular dynamics. J Mol Graph. 1996;14(1):33-38. doi:10.1016/02637855(96)00018-5

57. Appelt C, Wessolowski A, Dathe M, Schmieder P. Structures of cyclic, antimicrobial peptides in a membrane-mimicking environment define requirements for activity. J Peptide Sci. 2008;14(4):524-527. doi:10.1002/psc.924

58. Wessolowski A, Bienert M, Dathe M. Antimicrobial activity of arginine-and tryptophan-rich hexapeptides: the effects of aromatic clusters, d-amino acid substitution and cyclization. J Peptide Res. 2004;64(4):159-169. doi:10.1111/j.1399-3011.2004.00182.x

59. Los J, Kroes J, Albe K, Gordillo R, Katsnelson M, Fasolino A. Extended Tersoff potential for boron nitride: energetics and elastic properties of pristine and defective h-BN. Phys Rev B. 2017;96 (18):184108. doi:10.1103/PhysRevB.96.184108
60. Park S, Schulten K. Calculating potentials of mean force from steered molecular dynamics simulations. J Chem Phys. 2004;120 (13):5946-5961. doi:10.1063/1.1651473

61. Hirschfelder JO, Curtiss CF, Bird RB, Mayer MG. Molecular Theory of Gases and Liquids. Vol. 165. New York: Wiley; 1964.

62. Kang Y, Liu Y-C, Wang Q, Shen J-W, Wu T, Guan W-J. On the spontaneous encapsulation of proteins in carbon nanotubes. Biomaterials. 2009;30(14):2807-2815. doi:10.1016/j.biomateri als.2009.01.024

63. Gao H, Kong Y, Cui D, Ozkan CS. Spontaneous insertion of DNA oligonucleotides into carbon nanotubes. Nano Lett. 2003;3 (4):471-473. doi:10.1021/n1025967a

64. Kang Y, Wang Q, Liu Y-C, Wu T, Chen Q, Guan W-J. Dynamic mechanism of collagen-like peptide encapsulated into carbon nanotubes. J Phys Chem B. 2008;112(15):4801-4807. doi:10.1021/ jp711392g

65. Maleki R, Afrouzi $\mathrm{HH}$, Hosseini M, Toghraie D, Rostami S. Molecular dynamics simulation of Doxorubicin loading with $\mathrm{N}$-isopropyl acrylamide carbon nanotube in a drug delivery system. Comput Methods Programs Biomed. 2020;184:105303. doi:10.1016/ j.cmpb.2019.105303

66. Veclani D, Melchior A. Adsorption of ciprofloxacin on carbon nanotubes: insights from molecular dynamics simulations. J Mol Liq. 2020;298:111977. doi:10.1016/j.molliq.2019.111977

67. Lu D, Aksimentiev A, Shih AY, et al. The role of molecular modeling in bionanotechnology. Phys Biol. 2006;3(1):S40. doi:10.1088/14783975/3/1/S05

68. Zhang L, Peng G, Li J, et al. Molecular dynamics study on the configuration and arrangement of doxorubicin in carbon nanotubes. J Mol Liq. 2018;262:295-301. doi:10.1016/j.molliq.2018.04.097

69. Shen J-W, Wu T, Wang Q, Kang Y. Induced stepwise conformational change of human serum albumin on carbon nanotube surfaces. Biomaterials. 2008;29(28):3847-3855. doi:10.1016/j.biomateria 1s.2008.06.013
International Journal of Nanomedicine

\section{Publish your work in this journal}

The International Journal of Nanomedicine is an international, peerreviewed journal focusing on the application of nanotechnology in diagnostics, therapeutics, and drug delivery systems throughout the biomedical field. This journal is indexed on PubMed Central, MedLine, CAS, SciSearch ${ }^{\circledR}$, Current Contents ${ }^{\circledR} /$ Clinical Medicine,
Journal Citation Reports/Science Edition, EMBase, Scopus and the Elsevier Bibliographic databases. The manuscript management system is completely online and includes a very quick and fair peer-review system, which is all easy to use. Visit http://www.dovepress.com/ testimonials.php to read real quotes from published authors. 